\title{
The phylogenetic position of the zebrafish (Danio rerio), a model system in developmental biology: an invitation to the comparative method
}

\author{
AXEL MEYER, GHRISTIANE H. BIERMANN AND GUILLERMO ORTÍ \\ Department of Ecology and Evolution, State University of New York, Stony Brook, New York 11794-5245, U.S.A.
}

\section{SUMMARY}

The zebrafish, Danio (Brachydanio) rerio, has become one of the most widely studied model systems in developmental biology. We present a DNA-based phylogeny of zebrafish and other species of the genus Danio, and the genera Rasbora, Puntius and Cyprinus. Homologous regions of the large (16S) mitochondrial ribosomal RNA gene were amplified by the polymerase chain reaction and directly sequenced. The phylogeny revealed: (i) the zebrafish, Danio (Brachydanio) rerio, is identical in its $16 S$ sequence to its aquarium breeding morph, the leopard danio; (ii) the pearl danio (Danio albolineatus) is more closely related to the zebrafish than the giant danio (Danio aequipinnatus); and (iii) species of the genus Rasbora (hetermorpha, trilineata, elegans, pauciperforata, dorsiocellata) are more closely related to the danios than members of the genus Puntius (tetrazona, conchonius) and Cyprinus, the carp. All of these species are readily available in the aquarium trade, easily kept and bred in captivity, and amenable to developmental work. It is hoped that this molecular phylogeny will invite developmental biologists to use the comparative method to ask questions about function (e.g. cellular and genetic aspects) and evolution of zebrafish developmental biology in a phylogenetic context.

\section{INTRODUGTION}

The comparative method has enjoyed a long successful history in classical biology and also in developmental biology at the cellular and molecular level (Baldwin 1902; Waddington 1957; Gould 1977; Goodwin et al. 1983; Raff \& Kaufman 1983; Arthur 1984; for a recent review see Harvey \& Pagel 1991). It was Darwin's favourite method of research and remains the most widely used technique for answering questions about change and evolution in biology and the relation of development and evolution (Ghiselin 1984; and see references in Harvey \& Pagel 1991). A recent resurgence of interest in the comparative method involved finding techniques that are based on explicit evolutionary and statistical models (Brooks \& McLennan 1991; Harvey \& Pagel 1991). The aim of this approach is to study evolution of the phenotypic and genetic traits of organisms based on knowledge of the phylogenetic relations among the species compared. In developmental biology, comparisons tend to be made across large evolutionary distances, often between phyla, and focusing on leech, sea urchin, fruit fly, frog, chicken, mouse and rat. The new interest in the development of zebrafish adds this species to the list of vertebrate model systems.

Fine-grained (on a phylogenetic level) comparisons of developmental patterns among closely related species (e.g. mouse and rat) can provide insights that are not easily gained from comparisons among distantly related species. For example, the unexpectedly large differences in larval morphology of sea urchins suggests that a few molecular changes can produce strikingly different phenotypes (Strathmann et al. 1992; Raff 1992). Closely related species have genes in common because they share recent common ancestors; some of these genes might be absent or much modified among distant relatives. Homology at the phenotypic

Table 1. Systematic position and current scientific and common names of species in this study

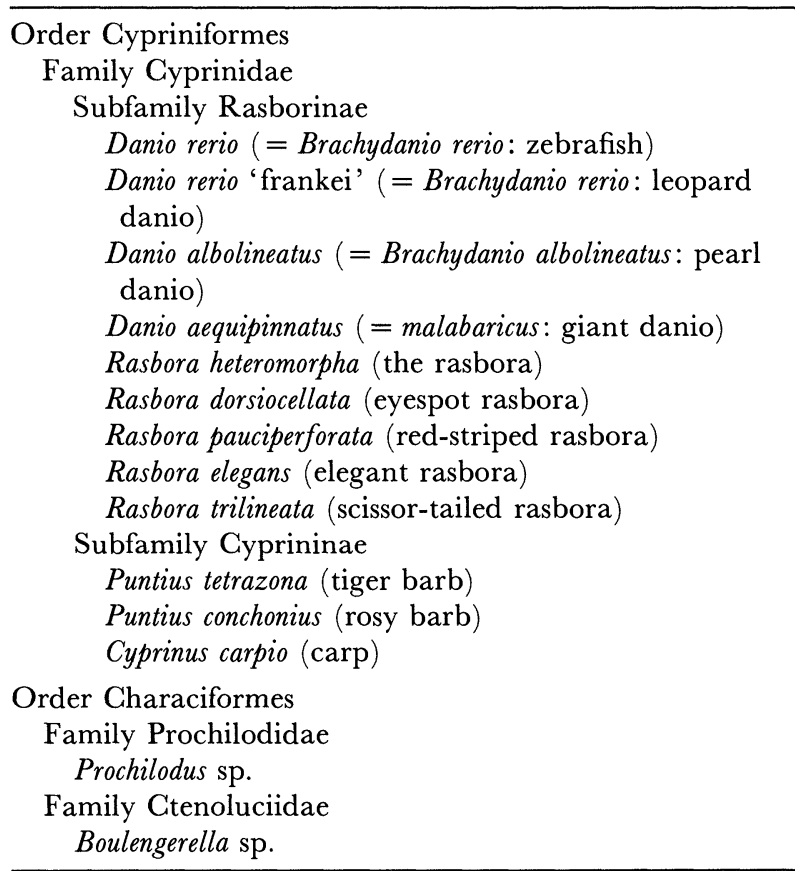




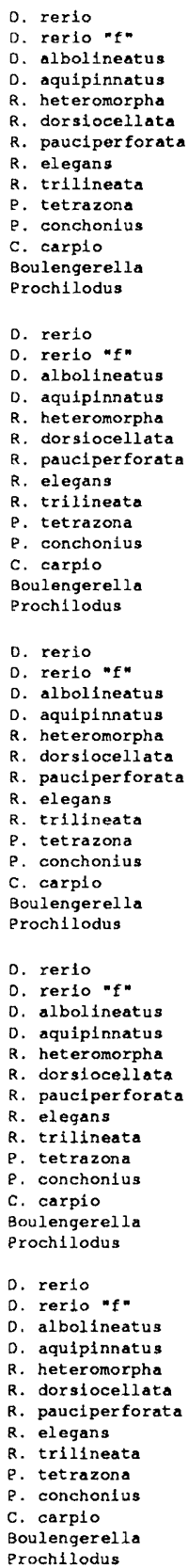

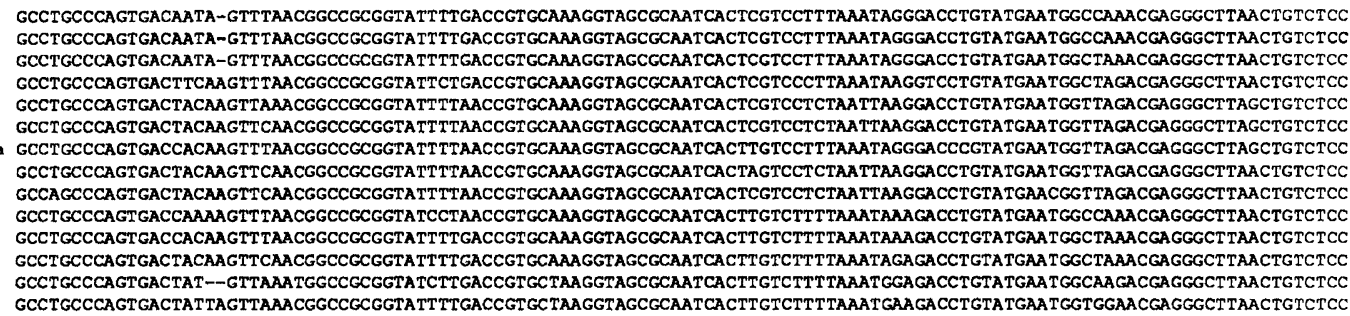

CCCATCAAGTCAGTGAAATTGATCTATCCGTGCAGAAGCGGATATAATAATACAAGACGAGAAGACCCTTTGGAGCTTAAGGTACAAGATTTAATTTACATCAAGCAAACC----AATAA [2 40] CCCATCAAGTCAGTGAAATTGATCTATCCGTGCAGAAGCGGATATAATAATACAAGACGAGAAGACCCTTTGGAGCTTAAGGTACAAGATTTAATTTACATCAAGCAAACC--D-AATAA CCACTCAAGTCAGTGAAATTGATTTACCCGTGCAGAAGCGGGTATGATAATACAAGACGAGAAGACCCTTTGGAGCTTAAGGTACAAGGTTTAAATTATACTAAGCTAGC------AATAA CCCTTCAGGTCAGTGAAATTGATCTATCCGTGCAGAAGCGGATATAAGAATACAAGACGAGAAGACCCTTTGGAGCTTTAGGTACAAGGCTTA-CTTACGTCAAATAATCT----AATCA CCCTTCCAGTCAGTGAAATTGATCTATCCGTGCAGAAGCGGGTATAATAATACAAGAGGAGAAGACCCTCTGGAGCTTAAGGTACAAGATCNNNNNNNNGTTAAACAAACC--CCA-TAA CCITTCCAGTCAGTGAAATT GATCTATCCGTGCAGAAGCGGGTA TAATAATACAAGACGAGAAGACCCTTTGGAGCTTAAGGTACA-GAAATCAATCATGTTAAACAAACC--CCA-TAA CITITCAGTCAGTGAAATTGATCTATCCGTGCAGAAGCGGGTATAATAATACAAGACGAGAAGACCCTTTGGAGCTTAAGGTACAAGA-CTCACCCATGTAAAACAAGTC--CTAATIA CTTTTCCAGTCAGT GAAAT TGATCTACCCGTGCAGAAGCGGGTGTAACAACACAAGACGAGAAGACCCTTTGGAGCTTAAGGTACAAGAA-TCAATTATGCTAAGCAAACT--TCAATAA CCTCTCCAGTCAGTGAAATTGATCTATCCGTGCAGAAGCGGGTATAATGATACAAGACGAGAAGACCCTTTGGAGCTTAAGGTACAAGAA-TCAATCATGTTAAACAAACT--TT--TAN CTTATCAAGTCAGTAAAATTGATCTACCCGTGCAGAAGCGGGTATAAACATACAAGACGAGAAGACCCTTTGGAGCTTAAGGTA-AAAA-TTCACCCACGTCAAGCAGCTTAATATACAA CATTTCCAGTCAGTGAAATTGATTTACCCGTGCAGAAGCGGGTATAAAAATACAAGACGAGAAGACCCTTTGGAGCTTAAGGTACAAAA-CTCAACCACGTCAAACAACTCTATAAA-CCTTTCAAGTCAGTGAAATT GATCTACCCGTGCAGAAGCGGGTATAATACTACAAGACGAGAAGACCCTTTGGAGCTTAAGGTACAAAA-CTCAACCACGTTAAGCAACTCAATAAA--A TCrTTT TGGTCAGTGAAATTGATCTACCCGTGCAGAAGCGGGTATAAGAATACAAGACGAGAAGACCCTT TGGAGCTTAAGACCTAAGA-CCAATCCCTGT TAAG-----TTATTAACTT

AAG------TGATTAAGCTAGTAAAAGATAAACCTCTACCTTCGGTTGGGGCGACCACGGAGTAAAAAACAACCTCCAAGCGGAACGGG--CACAGCC--CTAAAACCAAGA-GAA [360] AAG---1-TGATTAAACTAGTAAAAGA TAAACCTCTACCTTCGGTTGGGGCGACCACGGAGTAAAAAACAACCTCCAAGCGGAACGGG--CACAGCC--CTAAAACCAAGA-GAA

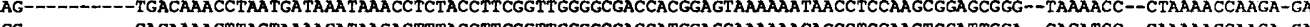

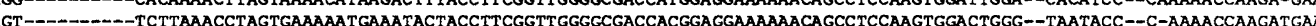

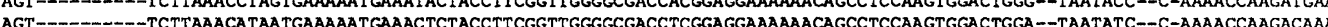
AGT---TCTTAAACATAATGAAAAAT TAAACTCTACCTTCGGTTGGGGCGACCTCGGAGGAAAAAACAGCCTCCAAGTGGACTGGA--TAATATC--C-AAAACCAAGACAAA AGT--------CCTTAAACCTAAT-AAAAATGAAACTCTACCTTCGGTTGGGGCGACCACGGAGAAAAAAATAACCTCCAAGTGGACTGGA--CAGTATC--C-AAAACCAAGA-AAA AGT----.--.-CCTTAAACCTAGTGAAAAA TGAAACTCTACCTTCGGTTGGGGCGACCACGGAGAAAAAAACAACCTCCAAGTGGACCGGA--TTATTTC--C-AAAACCAAGATAAA AGCAACTCCTTTATTATAAACCTTGTGGATTATGAAATAATACCTTCGGTTGGGGCGACCATGGAGAAAAGAAAACCTCCAAGTGGAATGAG--- ACCACCCCTCAAAACTAAGA-GAG AGCAACT---1--TAAACCTTGTGGCAATTGAGACTTTACCTTCGGTTGGGGCGACCACGGAGGAAAGTAAAGCCTCCAAGTGGATTAGG--GATAACCCCCTAAAACTAAGA-GAG AGCAA----AAACCTTGTGGATCATGAGATTTTACCTTCGGTTGGGGCGACCACGGAGGAAAGAAAAGCCTCCAGGTGGACTGGG--AA-AACCTCCTAAAACCAAGA-GAG AATAT----TAATAAAACAATTAA-GATACCTGG-CCTACGTCTTCGGTTGGGGCGACCACGG-GGAAAACAAAGCCCCCATGTGGAATGGG-GT TATAGACCCTAGATCCAAGA-GA AATGA------AATCAAACAAA-AC-GGCAACTGA-CCAGCGTCTTCGGTTGGGGCGACCACGG-GGAAAATGAAGCCCCCATGTGGAATGGG-GTAAT---CCCTAAAACTAAGA-GAG

ACATCTCTAAGCCTCAGAACACCTGACCAAAGACGATCCGGC---CTATAAGCCGATCAACGAACCAAGTTACCCTAGGGATAACAGCGCAATCCTCTCTAAGAGTCCATATCGACGAGG [480] ACATCTCTAAGCCTCAGAACACCTGACCAAAGACGATCCGGC---CTATAAGCCGATCAACGAACCAAGTTACCCTAGGGATAACAGCGCAATCCTCTCTAAGAGTCCATATCGACGAGC ACATCTCTAAGCCTCAGAACATCTGACCAAA-ACGATCCGGC---CTA-AAACCGATCAACGAACCAAGT TACCCTAGGGATAACAGCGCAATCCTCTTTAAGAGTCCATATCGACAAGG ACATCTCCACGTCACAGAACATCTGACCAATTATGATCCGGT---TAAAAAACCGATCAACGGACCAAGTTACCCTAGGGATAACAGCGCAATCCTCTCCAAGAGTCCATATCGACGAGG -CATATCTAAGTAACAGAACATCTGACCAAAAATGATCCGGC---CACAAA-CCGATCAACGAACCAAGTTACCCAAGGGATAACAGCGCAATCCTCTTCCAGAGTTCATATCGACGAGG - CACCTCTAAGTAACAGAACATCTGACCAAAAA TGATCCGGC---CATTAA-CCGATCAACGAACCAAGTTACCCAAGGGATAACAGCGCAATCCTCTTCCAGAGTT CATATCGACGAGG - CACCTCTAAGTAACAGAACATCTGACCAAATATGACCCGGC---CACAAAGCCGATCAACGAACTAAGTTACCCAAGGGATAACAGCGCAATCCCCTTCCAGAGTCCATATCGACGAGC ACAACTCTAAGTAACAGAACATCTGACCAAAAATGATCCGGC---CACAAG-CCGA TCAACGAACCAAGTTACCCAAGGGATAACAGCGCAATCCTCTTCCAGAGTTCATATCGACGAGA 作 ACATCT

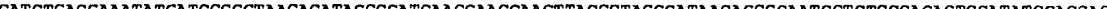
ACATCTCTAAGCCACAGACATCTGACCAAATATGATCCGGCTAACACATAGCCGA

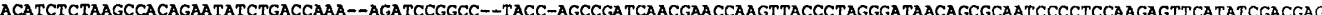

GGGTTTACGACCTCGATGTTGGATCAGGACATCCTAATGGTGCAGCCGCTATTAAGGG GGGTTTACGACCTCGATGTTGGATCAGGACATCCIAAT GGGTTTACGACCTCGATGTTGGATCAGGACATCCTAATCGIC GGGTTTACGACTCGATGTTGGATCAGGACATCCIAATGTGCAGCCGCTATTAAGG CTCGATGTTGGATCAGGACATCCIAATGGTGCAGCCGCTATTAGGGG CATGTGGATAGGACAY GGGTTTACGACCTCGATGTTGGATCAGGACATCCTAATGGTGCAGCCGCTATTAAGGC GGGTTTACGACCTCGATGTTGGATCAGGACATCCIAATGGTGCAGCCGCTATIAAGGG GGGTTTACGACCTCGATGTTGGA TCAGGACATCCTAATGGTGCAGCCGCTATTAAGGC GGGTTTACGACCTCGATGTTGGATCAGGACATCCTAATGGTGCAGCCGCTATTAAGGG GGGTTTACGACCTCGATGTTGGATCAGGACATCCTAATGGTGCAGCCGCTATTAAGGC GGGTTTACGACCTCGATGTTGGATCAGGACATCCTAATGGTGCAGCCGCTATTAAGGG

Figure 1. Aligned partial large mitochondrial ribosomal sequences of zebrafish and other species considered in this study. Species names can be inferred from table 1.

and genetic level (Patterson 1988) is often more problematic to assess among distantly related species, particularly for families of genes that underwent repeated duplication events, such as homeobox genes (see, for example, Scott \& Carroll 1987; Kappen et al. 1989; Runstadler \& Kocher 1991; Murtha et al. 1991; Krauss et al. 1992; Marx 1992; Tabin 1992; Schubert et al. 1993). Homology of genes among distant species, such as between Drosophila and zebrafish, is often ambiguous. It would appear that much insight into the functional aspects (both cellular and molecular) of development could be gained if several closely related species were compared, making the search for and identification of homologous genes simpler. Potential inferences about genotype-phenotype relations are difficult to test, but may be more tractable in a set of comparisons among closely related species whose evolutionary relations are known (Raff 1992).
The zebrafish, Danio (Brachydanio) rerio HamiltonBuchanan 1822, is one of the most widely used model systems in the developmental biology of lower vertebrates. Since the pioneering work of George Streisinger, literally hundreds of publications on zebrafish have appeared (Westerfield 1989), and knowledge about cellular and molecular aspects of zebrafish development continues to increase very rapidly. Part of the reason for the success of the zebrafish as a model system is that they are readily available in pet stores throughout the world and are easy to breed in captivity, which ensures a steady supply of embryos. Many of the advantages of zebrafish are also present in closely related species of fish, providing ideal conditions for a comparative approach.

The zebrafish Danio ('Brachydanio') rerio belongs to the family Cyprinidae in the order Cypriniformes. This family of fish contains about 1600 species (Nelson 
1984) and is one of the most successful families in terms of species number and geographic distribution. Several genera in addition to Danio are assigned to the subfamily Rasborinae (table 1), many of which are common in India, Pakistan, Nepal, Bangladesh, Sri Lanka, Thailand, the Malay Peninsula, Sumatra and the Yunnan province of China (Barman 1991). The genus Danio was first described by Hamilton-Buchanan in 1822. Weber and de Beaufort (1916) first proposed Brachydanio as subgenus of Danio. Since then, the classification of these genera has been reviewed repeatedly (see, for example, Hora \& Mukerji 1934), most recently by Barman (1991) who eliminated the genus Brachydanio by synonymizing with it Danio. Danio is therefore the valid genus name for the zebrafish $(D$. rerio) as well as for the pearl danio (D. albolineatus). This recent revision also found Danio malabaricus Jerdon 1849 to be synonymous with $D$. aequipinnatus McGlelland 1839, the latter name having precedence. Despite this taxonomic work, the phylogeny of danios and their phylogenetic relations to other cyprinid fishes remained untested.

Traditionally, systematists have used phenotypic characters to establish classifications and phylogenetic relations among species. Recent molecular approaches, namely the phylogenetic analysis of homologous DNA sequences to establish phylogenetic relations, have provided a successful alternative approach. Mitochondrial DNA in particular has been widely used to establish relationships among closely related species (see, for example, Meyer $1993 b$ ). We analysed partial sequences of the large mitochondrial ribosomal RNA gene to determine the phylogenetic position of zebrafish in relation to other species in its genus and several other genera of cyprinid fishes.

The sequences reported in this paper have been deposited in the GenBank database under accession numbers L14487-L14499.

\section{MATERIALS AND METHODS}

Table 1 lists the species used in this study and their current classification. Specimens were commercially obtained from several localities (Berkeley, Albany and San Francisco in California; Long Island in New York; and Lübeck in Germany) to survey potential intraspecific variation. Two specimens of zebrafish (WT, AB lines) were also obtained from the Kimmel laboratory at the University of Oregon. Four specimens of $D$. rerio, three specimens of $D$. rerio 'frankei', the aquarium breeding morph of the zebrafish, three specimens of $D$. albolineatus, and two specimens of $D$. aequipinnatus (= malabaricus) were sequenced. For the other species, one specimen per species was sequenced. The $16 S$ sequence of the carp (Cyprinus carpio) was obtained from GenBank (accession no. X61010). Two distantly related tetras (order Characiformes) of the genera Prochilodus and Boulengerella were used as outgroups in our phylogenetic analysis.

Specimens were preserved in $70 \%$ (by volume) ethanol or frozen, and total genomic DNA was extracted from white muscle or liver tissue by Proteinase K/SDS dissolution and purified by phenol-chloroform extraction and ethanol precipitation (Kocher et al. 1989; Sambrook et al. 1989). The polymerase chain reaction (PGR) (Saiki et al. 1988) was used to amplify a segment of the large (16S) mitochondrial ribosomal gene. Double-stranded amplifications were done in $25 \mu \mathrm{l}$ volumes containing (in millimoles per litre): 67 Tris (pH 8.8), $6.7 \mathrm{MgCl}_{2}, \quad 16.6\left(\mathrm{NH}_{4}\right)_{2} \mathrm{SO}_{4}, \quad 10$ 2-mercaptoethanol, 1 of each dNTP, $1 \mu \mathrm{m}$ of each primer, $10-1000 \mathrm{ng}$ genomic DNA, and 0.5 units of Taq Polymerase (Cetus Corp.). Primers used were 16Sar-L (5'-CGCGTGTTTATCAAAAACAT-3') (Palumbi et al. 1991) and 16Sbr-H (5'CGGGTCTGAACTCAGATCACGT-3') (Palumbi et al. 1991) to obtain an amplification product about 600 base pairs (b.p.) long. Gel purification (2.5\% NuSieve-Agarose, in TAE buffer) of the double-stranded product was followed by generation of single-stranded DNA of both strands for direct sequencing, by using asymmetric PGR (Gyllensten \& Erlich 1988). Single-stranded DNA was concentrated and desalted in spin columns (Millipore: Ultrafree-MC 30000), and both strands were sequenced by the dideoxy method using a commercial kit (Sequenase, United States Biochemical).

The orthologous DNA sequences obtained were aligned, using default settings, by clustal (Higgins \& Sharp 1988) and with a multiple sequence editor (ESEE: Cabot \& Beckenbach 1989). Parsimony analyses were done by using maximum parismony (MP) (PAUP: Swofford 1991). In PAUP, minimal trees were found with branch-and-bound, and bootstrap analyses ( 100 replications) were done with heuristic searches by using simple stepwise addition, the TBR branchswapping algorithm and the MULPARs option. Indels were coded as single characters irrespective of their length. When indels of different length overlapped, each size class of indels was assigned a particular character state. Parsimony analyses were done treating characters as unordered and assigning different weights to transitions, transversion and indels; e.g. treating all kinds of substitutions equally, transversions and indels three times the weight of transitions, or transversions only.

Maximum likelihood analysis (ML) (Felsenstein 1981, 1991) was done using the PHYLIP package (DNAML Version 3.4) using default parameters: empirical base frequencies and a transition/transversion ratio of 2 .

Neighbour-joining analysis (NJ) (Saitou \& Nei 1987) was done with the PHYLIP package (NEIGHBOR, Version 3.4) (Felsenstein 1991). Distances were computed by using DNADIST with a maximum-likelihood multiple-substitution correction. Bootstrapping with 100 replications (Felsenstein 1985) was done with SEQBOOT in PHYLIP to estimate confidence in the phylogenetic trees.

\section{RESULTS}

Figure 1 shows the aligned sequences of the 14 species used in this study. Up to 538 b.p. (including indels) were determined for each species. This portion of the $16 S$ gene corresponds about to positions 2510 to 3080 in the human complete mitochondrial DNA sequence (Anderson et al. 1981). We found no intraspecific differences in $D$. albolineatus and $D$. aequipinnatus, and only one transition among the four specimens of $D$. rerio and three specimens of $D$. rerio 'frankei' (the spotted aquarium breeding morph called the leopard danio) (position 261; figure 1). This position varied within $D$. rerio proper and within the leopard danio, supporting the notion that the leopard danio (figure 2) is not distinct from $D$. rerio.

The observed substitution pattern conforms to expectations based on prior knowledge about the evolutionary behaviour of this molecule (reviewed in 


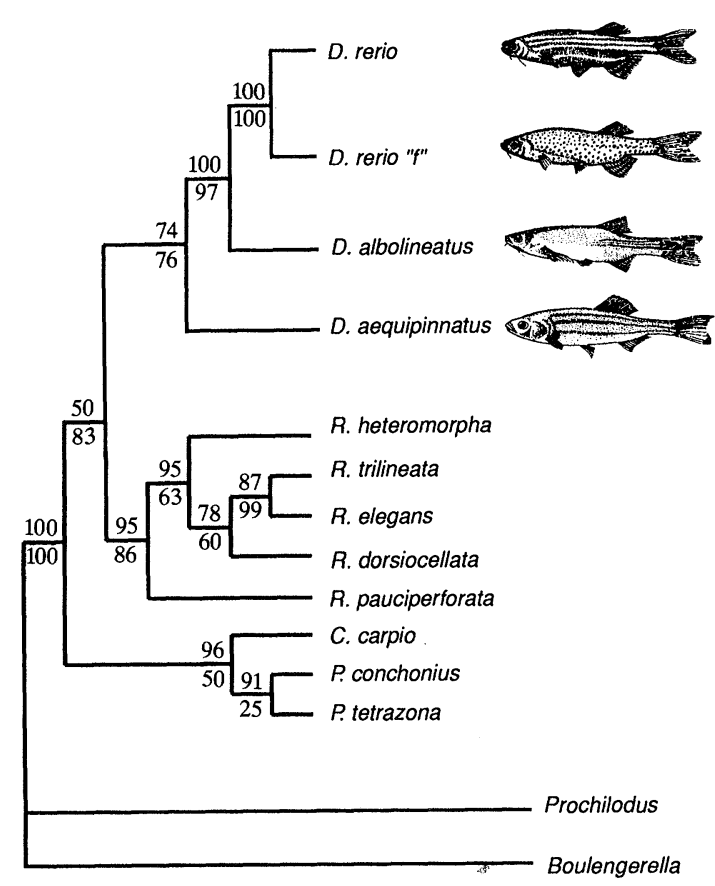

Figure 2. Molecular phylogenetic position of the zebrafish. The same cladogram was inferred by the neighbour-joining method (Saitou \& Nei 1987), maximum-likelihood (Felsenstein 1981) and maximum-parsimony methods (PAUP: Swofford 1991). Maximum likelihood confirmed that all branches are significantly positive (at $p<0.01$ ). The maximum-parsimony method (using no differential weights for transitions and transversions, calculated with PAUP (Swofford 1991)) found four equally parsimonius trees (length $=438$ steps, consistency index $(\mathrm{CI})=0.662$, cI excluding uninformative characters $=0.583$ ). One of these trees is the topology shown, the other three varied in the relationships among the Puntius and Cyprinus and the parsimony bootstrap tree did not resolve (at the $50 \%$ level) the relationships among Puntius and Cyprinus. The numbers above the branches are bootstrap values (Felsenstein 1985) using the $\mathrm{NJ}$, and those below are from the maximum parsimony bootstrap analyses.

Meyer $1993 a$ ). Single base pair substitutions are the most frequently observed type of change, whereas indels are much less frequent, as expected from other studies of mitochondrial DNA in fish (reviewed in Meyer 1993a). Indels are more frequent in inferred loops than in stem regions (reviewed in Mindell \& Honeycutt 1990, for example; Meyer 1993a), potentially retaining the secondary structure of the molecule. As is typical for mitochondrial DNA (Brown et al. 1982; reviewed in Meyer 1993a,b), transition substitutions outnumber transversions among closely related species. For example, D. rerio and D. albolineatus differ by a total of 33 substitutions, of which $25(76 \%)$ are transitions and eight $(24 \%)$ are transversions (table 2). As expected, transversions become more abundant between more distantly related species (e.g. carp and zebrafish: $36 \%$ of 65 changes were transversions).

The molecular phylogeny obtained agrees with the current classification (table 1 ; figure 2). The molecular tree (figure 2) is corroborated by all three phylogenetic methods used, and most branches are supported with high confidence (Felsenstein 1985), as indicated by the high bootstrap values. All variations of the parsimony analysis yielded congruent topologies with the one shown, except for the relationship between Puntius and Cyprinus which remains tentative. But the neighbourjoining and maximum-likelihood methods strongly favour the grouping shown (figure 2).

The phylogeny (figure 2) derived from the $16 S$ sequences supports the sister group relationship of $D$. rerio and $D$. albolineatus, in agreement with their previous (before Barman 1991) placement into the now obsolete genus Brachydanio. Of the species included in this study, the giant danio is the most closely related species to these two. All species of the Danio and all species of Rasbora are placed into two distinct clades. The $16 S$ phylogeny supports the classification of these two genera into the subfamily Rasborinae, and the position of the two genera Cyrpinus and Puntius into the subfamily Gyprininae.

\section{DISGUSSION}

Biologists striving to understand what has shaped biological phenomena need to know the past to make

\section{Table 2. Matrix of sequence differences of partial $16 \mathrm{~S}$ mitochondrial DNA}

(Values above the diagonal are uncorrected percent sequence difference including substitutions and indels, below are the total numbers of substitutions and transversions only.)

\begin{tabular}{|c|c|c|c|c|c|c|c|c|c|c|c|c|c|c|c|}
\hline & & 1 & 2 & 3 & 4 & 5 & 6 & 7 & 8 & 9 & 10 & 11 & 12 & 13 & 14 \\
\hline 1 & oulengerella & 一 & 13.4 & 15.3 & 17.6 & 17.4 & 16.7 & 19.5 & 17.8 & 19.4 & 17.4 & 18.6 & 18.9 & 18.5 & 16.8 \\
\hline 2 & Prochilodus & $72 / 19$ & - & 6.6 & 19.2 & .0 & 17.9 & .7 & 16.7 & 9.2 & 7.2 & 8.3 & 8.8 & 17.8 & 15.6 \\
\hline 3 & C. carpio & $82 / 38$ & $88 / 34$ & - & 12.1 & 12.1 & 12.4 & 12.7 & 10.1 & 12.4 & 10.8 & 11.8 & 11.6 & 9.5 & 5.7 \\
\hline 4 & D. rerio & $94 / 36$ & $101 / 32$ & $65 / 24$ & - & 0.2 & 6.1 & 11.0 & 11.3 & 13.1 & 13.8 & 13.0 & 13.1 & 13.8 & 13.4 \\
\hline 5 & D. rerio ' $f$ ' & $93 / 36$ & $100 / 32$ & $65 / 24$ & $1 / 0$ & - & 6.1 & 11.0 & 11.3 & 13.1 & 13.8 & 13.0 & 13.1 & 13.8 & 13.4 \\
\hline 6 & D. albolineatus & $89 / 34$ & $94 / 29$ & $66 / 21$ & $33 / 8$ & $33 / 8$ & - & 13.1 & 11.5 & 12.3 & 13.4 & 12.5 & 11.9 & 15.6 & 13.5 \\
\hline 7 & D. aequipinnatus & $104 / 39$ & $109 / 37$ & $68 / 20$ & $60 / 24$ & $60 / 24$ & $71 / 24$ & - & 11.8 & 13.3 & 13.7 & 13.1 & 13.1 & 16.1 & 14.0 \\
\hline 8 & R. heteromorpha & $95 / 37$ & $88 / 29$ & $54 / 17$ & $61 / 24$ & $61 / 24$ & $62 / 22$ & $64 / 25$ & - & 5.5 & 7.4 & 3.5 & 6.5 & 14.6 & 11.9 \\
\hline 9 & R. trilineata & $103 / 40$ & $101 / 33$ & $66 / 17$ & $71 / 27$ & $71 / 27$ & $66 / 24$ & $72 / 26$ & $30 / 7$ & - & 9.4 & 4.8 & 4.8 & 15.9 & 14.0 \\
\hline 10 & R. pauciperforata & $93 / 37$ & $91 / 33$ & $58 / 16$ & $75 / 23$ & $75 / 23$ & $72 / 23$ & $74 / 22$ & $40 / 10$ & $51 / 11$ & - & 7.0 & 9.2 & 14.5 & 10.8 \\
\hline 11 & R. dorsiocellata & $99 / 41$ & $96 / 31$ & $63 / 18$ & $70 / 26$ & $70 / 26$ & $67 / 24$ & $71 / 29$ & $19 / 8$ & $26 / 6$ & $38 / 12$ & - & 5.5 & 16.7 & 13.3 \\
\hline 12 & R. elegans & $101 / 41$ & $99 / 33$ & $62 / 16$ & $71 / 26$ & $71 / 26$ & $64 / 23$ & $71 / 27$ & $35 / 6$ & $26 / 5$ & $50 / 10$ & $30 / 5$ & 一 & 14.8 & 13.2 \\
\hline 13 & P. tetrazona & $101 / 42$ & $96 / 34$ & $52 / 18$ & $75 / 25$ & $75 / 25$ & $84 / 28$ & $87 / 29$ & $79 / 27$ & $86 / 29$ & $79 / 27$ & $90 / 31$ & $80 / 29$ & - & 9.7 \\
\hline 14 & P. conchorius & $90 / 33$ & $83 / 29$ & $31 / 12$ & $72 / 26$ & $72 / 26$ & $72 / 23$ & $75 / 25$ & $64 / 20$ & $75 / 20$ & $58 / 18$ & $71 / 20$ & $71 / 19$ & $53 / 22$ & - \\
\hline
\end{tabular}


sense of the present. Phylogenies are the necessary first step in the comparative method, and can be used to distinguish between alternative hypotheses and competing models of evolution. Usually, species that share a recent common ancestor tend to be similar in their genes, their phenotypes, and probably in their development (but see del Pino \& Elinson 1983; Wray \& Raff 1991; Raff 1992). However, distantly related species that live in the same environment and experience the same selection pressures might evolve similar adapted phenotypes convergently. This is why the phenotype can be a misleading indicator of evolutionary relationships.

Many examples show that the evolution of developmental mechanisms and adult phenotypes do not proceed at a regular pace and that large morphological differences may be caused by small genetic differences. Hence, to infer descent and phylogeny from the phenotype can be misleading because the rate of morphological evolution can be so variable. Molecules tend to be more impartial tracers of evolutionary relationships. Murray (1989) demonstrated that a change in a single parameter of the reaction diffusion mechanism (e.g. for coat coloration in mammals) may be all that is required for switches between stripes and spots. The coloration of the striped zebrafish and one of its breeding morphs, the spotted leopard danio, might be brought about by similarly small genetic differences. Comparisons between closely related species allow us to test the generality of biological phenomena. An example where development shows much variation among closely related species involves the plasticity of the mode of development in many marine invertebrates and ascidians. Even in sister species of echinoderms the pronounced differences found between some larval forms, which start at the fourth cleavage, indicate that morphogenetic mechanisms of early embryonic stages can undergo drastic changes within short geological time spans (Wray \& Raff 1991). Based on current knowledge of echinoderm phylogeny, direct development has evolved independently from a specialized feeding pluteus in at least six clades in sea urchins accompanied by analogous changes in gene expression (Wray \& Raff 1991; Wray 1992). Some aquatic vertebrates, too, exhibit modified early development, e.g. hylid frogs (del Pino \& Elinson 1983). Ballard (1981) pointed out the need for studies on the early development of more vertebrate species whose evolutionary relationships have been established by independent criteria.

In development (as in most fields of biology) strong inferences about a large number of species tend to be made from a relatively small number of model systems. There are very good practical reasons for this. The ground work necessary to establish a new model system and to start studies of the cellular and molecular genetic aspects of development are so prohibitive that most laboratories will not venture to do so. The practical advantages of zebrafish as a model system that have made it so popular in developmental biology (e.g. they are easily obtained and bred, and have clear eggs), apply to many other closely related species of fishes. Our phylogeny of the zebrafish is meant as an invitation to study the biology of cyprinid fishes in a fine-grained evolutionary framework. When comparing zebrafish development with several other closely related species meaningful insights will surely be gained into the generality of zebrafish developmental biology and the evolution of development.

We are grateful to the National Science Foundation, U.S.A., for providing partial support for this study (grants BSR9107838 and BSR-9119867 to A.M.), to Gharlene Walker from the Kimmel laboratory (University of Oregon) for kindly providing us with some zebrafish, to J. Nelson for pointing out literature on zebrafish classification, to Catherine Sexton for the drawings in figure 2, and to Christian Sturmbauer, Paul Wilson, Gregory Wray and two anonymous referees for comments on the manuscript.

\section{REFERENCES}

Anderson, S., Bankier, A. T., Barrell, B. G., de Bruijn, M. H. L., Goulson, A. R., Drouin, J., Eperon, I. G., Nierlich, D. P., Roe, B. A., Sanger, F., Schreier, P. H., Smith, A. J. H., Staden, R. \& Young, I. G. 1981 Sequence and organization of the human mitochondrial genome. Nature, Lond. 290, 457-465.

Arthur, W. 1984 Mechanisms of morphological evolution. Chichester: John Wiley.

Baldwin, J. W. 1902 Development and evolution. New York: Macmillan.

Ballard, W. W. 1981 Morphogenetic movements and fate maps of vertebrates. Am. Zool. 21, 391-399.

Barman, R. P. 1991 A taxonomic revision of the IndoBurmese species of Danio Hamilton-Buchanan (Pisces: Cyprinidae). Rec. zool. Surv. India occ. Paper 137, 1-91.

Brooks, D. R. \& McLennan, D. A. 1991 Phylogeny, ecology, and behavior: A research program in comparative biology. Chicago University Press.

Brown, W. M., Prager, E. M., Wang, A. \& Wilson, A. C. 1982 Mitochondrial DNA sequences of primates: tempo and mode of evolution. J. molec. Evol. 18, 225-239.

Cabot, E. L. \& Beckenbach, A. T. 1989 Simultaneous editing of multiple nucleic acid and protein sequences with ESEE. CABIOS 5, 233-234.

del Pino, E. M. \& Elinson, R. P. 1983 A novel developmental pattern for frogs: gastrulation produces an embryonic disk. Nature, Lond. 306, 589-591.

Felsenstein, J. 1981 Evolutionary trees from DNA sequences: A maximum likelihood approach. J. molec. Evol. 17, 368-376.

Felsenstein, J. 1985 Confidence limits on phylogenies: an approach using the bootstrap. Evolution 39, 783-791.

Felsenstein, J. 1991 PHYLIP (Phylogenetic Inference Package), version 3.42. Seattle: University of Washington.

Ghiselin, M. T. 1984 The triumph of the Darwinian method. University of Chicago Press.

Goodwin, B. C., Holder, N. \& Wylie, G. C. (ed.) 1983 Development and evolution. Cambridge University Press.

Gould, S. J. 1977 Ontogeny and phylogeny. Cambridge, Massachusetts: Harvard University Press.

Gyllensten, U. B. \& Erlich, H. A. 1988 Generation of single stranded DNA by the polymerase chain reaction and its application to direct sequencing of the HLA-DQA locus. Proc. natn. Acad. Sci. U.S.A. 85, 7652-7655.

Hamilton-Buchanan, F. 1822 An account of the fishes found in the river Ganges and its branches. Edinburgh: Archibald Constable.

Harvey, P. H. \& Pagel, M. D. 1991 The comparative method in evolutionary biology. Oxford University Press. 
Higgins, D. G. \& Sharp, P. M. 1988 clustal: a package for performing multiple sequence alignment on a microcomputer. Gene 73, 237-244.

Hora, S. L. \& Mukerji, D. D. 1934 Notes on fishes in the Indian Museum. XXII. On a collection of fish from the Shan States and the Pegu Yomas, Burma. Rec. Indian Mus. 36, 123-138.

Kappen, G., Schughart, K. \& Ruddle, F. H. 1989 Two steps in the evolution of Antennapedia-class vertebrate homeobox genes. Proc. natn. Acad. Sci. U.S.A. 86, 5459-5463.

Kocher, T. D., Thomas, W. K., Meyer, A., Edwards, S. V., Pääbo, S., Villablanca, F. X. \& Wilson, A. C. 1989 Dynamics of mitochondrial DNA evolution in animals. Proc. natn. Acad. Sci. U.S.A. 86, 6196-6200.

Krauss, S., Maden, M., Holder, N. \& Wilson, S. W. 1992 Zebrafish pax [b] is involved in the formation of the midbrain-hindbrain boundary. Nature, Lond. 360, 87-89.

Marx, J. 1992 Homeobox genes go evolutionary. Science, Wash. 255, 399-401.

Meyer, A. 1993 a Evolution of mitochondrial DNA in fish. In Biochemistry and molecular biology of fishes, vol. 2 (ed. P. W. Hochachka \& T. P. Mommsen). Amsterdam: Elsevier. (In the press.)

Meyer, A. $1993 b$ Molecular phylogenetic studies of fish. In Genetics and evolution of aquatic organisms (ed. A. Beaumont). London: Chapman and Hall. (In the press.)

Mindell, D. P. \& Honeycutt, R. L. 1990 Ribosomal RNA in vertebrates: Evolution and phylogenetic applications. A. Rev. Ecol. Syst. 21, 541-566.

Murray, J. D. 1989 Mathematical biology. Berlin: Springer Verlag.

Murtha, M. T., Leckman, J. F. \& Ruddle, F. H. 1991 Detection of homeobox genes in development and evolution. Proc. natn. Acad. Sci. U.S.A. 88, 10711-10715.

Nelson, J. S. 1984 Fishes of the world, 2nd edn. New York: Wiley.

Palumbi, S. R., Martin, A., Romano, S., McMillan, W. O., Stice, L. \& Grabowski, G. 1991 The simple fool's guide to PCR. Honolulu: University of Hawaii Press.

Patterson, C. 1988 Homology in classical and molecular biology. Molec. Biol. Evol. 5, 603-625.

Raff, R. A. 1992 Direct-developing sea urchins and the evolutionary reorganization of early development. BioEssays 14, 211-218.
Raff, R. A. \& Kaufman, T. C. 1983 Embryos, genes, and evolution. The developmental-genetic basis of evolutionary change. New York: Macmillan.

Runstadler, J. A. \& Kocher, T. D. 1991 A new antennapediaclass gene from the zebrafish. Nucl. Acids Res. 19, 5434.

Saiki, R. K., Gelfand, D. H., Stoffel, S., Scharf, S., Higuchi, R., Horn, R., Mullis, K. B. \& Erlich, H. A. 1988 Primerdirected enzymatic amplification of DNA with a thermostable DNA polymerase. Science, Wash. 239, 487-491.

Saitou, H. \& Nei, M. 1987 The neighbor-joining method: a new method for reconstructing phylogenetic trees. Molec. Biol. Evol. 4, 406-425.

Sambrook, J., Fritsch, E. F. \& Maniatis, T. 1989 Molecular cloning : a laboratory manual. New York: Cold Spring Harbor Laboratory Press.

Schubert, F. R., Nieselt-Struwe, K. \& Gruss, P. 1993 The antennapedia-type homeobox genes have evolved from three percursors separated early in metazoan evolution. Proc. natn. Acad. Sci. U.S.A. 90, 143-147.

Scott, M. P. \& Carroll, S. B. 1987 The segmentation and homeotic gene network in early Drosophila development. Cell 51, 689-698.

Strathmann, R. R., Fenaux, L. \& Strathmann, M. F. 1992 Heterochronic developmental plasticity in larval sea urchins and its implications for evolution of nonfeeding larvae. Evolution 46, 972-986.

Swofford, D. L. 1991 Phylogenetic analysis using parsimony (PAUP Version 3.0s). Champaign: Illinois Natural History Survey.

Tabin, C. J. 1992 Why we have (only) five fingers per hand: Hox genes and the evolution of paired limbs. Development 116, 289-296.

Waddington, C. H. 1957 The strategy of genes. London: Allen and Unwin.

Weber, M. \& de Beaufort, L. F. 1916 The fishes of the indoAustralian Archipelago. Leiden: E. J. Brill.

Westerfield, M. 1989 The zebrafish book: a guide for the laboratory use of zebrafish (Brachdanio rerio). Eugene: University of Oregon Press.

Wray, G. A. \& Raff, R. A. 1991 The evolution of developmental strategy in marine invertebrates. Trends Ecol. Evol. 6, 45-50.

Wray, G. A. 1992 Rates of evolution in developmental processes. Am. Zool. 32, 123-134.

Received 23 March 1993; accepted 5 April 1993 